\title{
Ubiquitination of System Xc- and GPX4 in rat brain stimulates ferroptosis and inflammation in hippocampal neurons after subarachnoid hemorrhage
}

Junhui Chen

Wuhan University Renmin Hospital

Chunlei Zhang

904th Hospital of Joint Logistic Support force of PLA

Yuhai Wang

904th Hospital of Joint Logistic Support Force of PLA

Zhuanghua Liu

904th Hospital of oint Logistic Surpport Force of PLA

\section{Shuo Yang}

904th Hospital of Joint Logistic Support Force of PLA

\section{Shoumeng Han}

Wuhan University Renmin Hospital

Qi Tian

Wuhan University Renmin Hospital

Mingchang Li

Wuhan University Renmin Hospital

Qianxue Chen ( $\square$ Qianxuech1030@163.com )

Wuhan University Renmin Hospital

\section{Research}

Keywords: Subarachnoid hemorrhage, Ferroptosis, System Xc-, GPX4, Ubiquitination, Hippocampal neuron

Posted Date: July 24th, 2020

DOI: https://doi.org/10.21203/rs.3.rs-41482/v1

License: (9) (1) This work is licensed under a Creative Commons Attribution 4.0 International License. Read Full License 


\section{Abstract}

Background: Ferroptosis is a peroxidation-driven form of cell death. Glutathione peroxidase 4 (GPX4) and System $\mathrm{Xc}^{-}$are leading regulators of ferroptosis. This study investigated System $\mathrm{Xc}^{-}$and GPX4 ubiquitination in ferroptosis after subarachnoid hemorrhage (SAH).

Methods: SAH rat model was established by artery perforation operation. The mortality, neurological score, brain water content and blood brain barrier permeability were and analyzed. The morphology of mitochondria was observed, the content of reactive oxygen species and the pathology of hippocampal neurons were evaluated, and the iron deposition was observed. Levels of ferroptosis-related proteins and proinflammatory factors were detected. The protein interaction between System $\mathrm{Xc}^{-}$and GPX4 was verified. After ferroptosis was induced by FIN56 in neurons, the neurons were transfected with OTUB1 overexpression vector or ubiquitination inhibitors to verify the regulatory mechanism of System $\mathrm{Xc}^{-}$and GPX4 ubiquitination in ferroptosis.

Results: After SAH operation, GPX4 was downregulated, and induced iron deposition and ROS accumulation. In SAH rats, System $\mathrm{Xc}^{-}$function was lost, GSH decreased, MDA content and SLC3A2, SLC7A11 and GPX4 ubiquitination increased, and OTUB1 expression decreased. SLC3A2 and OTUB1, GPX4 and OTUB1 can interact with each other in protein. Overexpression of OTUB1 reduced the ubiquitination of SLC3A2, SLC7A11 and GPX4 in FIN56-induced ferroptosis in neurons. Ubiquitination inhibitor or OTUB1 increased GSH level in ferroptotic neurons, and reduced ROS accumulation and inflammatory damage.

Conclusion: SAH induced the ubiquitination of System $\mathrm{Xc}^{-}$and GPX4, resulting in GSH depletion, increased lipid oxidative damage, and promoted ferroptosis in hippocampal neurons.

\section{Background}

Subarachnoid hemorrhage (SAH) commonly presents with an acute headache and neurological symptoms, including cranial neuropathies, focal weakness and meningism [1]. SAH often occurs after the rupture of cerebral aneurysm, resulting into bleeding as well as clot formation [2]. Nearly $15 \%$ of SAH patients die before arriving at the hospital, $25 \%$ die within 24 hours and $45 \%$ within 30 days, and only $1 / 3$ patients recover completely after treatment [3]. The foremost risk factors for SAH are mainly hypertension, old age, heavy drinking and smoking, and the most serious complication after SAH is cerebral vasospasm [2]. Noncontrast head computed tomography performed within 6 hours is reliable for SAH diagnosis, but after 6 hours lumbar puncture or computed tomography angiography may be needed [3]. Despite significant progress in surgical techniques, radiology and anesthesiology, the mortality and incidence rates after spontaneous SAH in recent years have not witnessed desirable reduction [4]. Therefore, it is paramount to identify the molecular mechanism of SAH and to develop more effective target treatments. 
Like the organism, the cells die in different patterns, including apoptosis, necrosis, autophagy, entosis, ferroptosis, and lysosome-dependent cell death [5]. Ferroptosis is an iron-dependent cell death initiated and progressed by lipid peroxide accumulation [5] and reactive oxygen species (ROS) production [6]. Ferroptosis is implicated in various diseases, including neurodegenerative diseases, acute renal failure and cancers [7]. Ferroptosis is featured with mitochondrial damage, inflammation and detrimental lipid ROS formation [8], which are also the main manifestation after SAH [9]. So it is rational to suspect that lipid ROS formation and neuroinflammation in hippocampal neurons after SAH are caused by ferroptosis. Additionally, ferroptosis is specifically activated by missing glutathione peroxidase 4 (GPX4) activities [10]. GPX4, an antioxidant defense enzyme is functional to repair oxidative damage to lipids and is a leading inhibitor of ferroptosis [11]. System $\mathrm{Xc}^{-}$is composed of SLC7A11 and SLC3A2 which imports the extracellular oxidized form of cysteine for intracellular glutamate antiporter [12]. A ferroptosis inducer erastin inactivates GPX enzymes, depletes glutathione (GSH) through preventing cystine uptake via inhibition of System $\mathrm{Xc}^{-}$, and induces ROS accumulation and causes ferroptosis [7]. However, the exact regulation mechanisms of System $\mathrm{Xc}^{-}$and GPX4 in the process of ferroptosis in hippocampal neurons after SAH are not yet studied. The comprehensive in vivo and in vitro experiments were conducted in this study to testify the involvement of System $\mathrm{Xc}^{-}$and GPX4 in ferroptosis-induced neuron injury after SAH, which may provide novel insights for therapeutic interventions in the future.

\section{Materials And Methods}

\subsection{Experimental animals}

All experimental adult Sprague Dawley rats $(280-350 \mathrm{~g})$ were obtained from the Experimental Animal Center of Shanghai Jiaotong University (license number SYXK (Shanghai) 2018-0028). The rats were raised in a specific pathogen-free laboratory at suitable temperature, with a 12-h light/dark cycle, proper humidity and free access to food and water. All surgical operations were performed under deep anesthesia to minimize the pain of animals.

A total of 126 rats were assigned to sham group $(n=48)$ and SAH group $(n=78)$. Nine of them died during or immediately after the operation, and 11 of them were excluded because of their low SAH scores. At 24 and 72 hours after operation, rats were euthanized by intraperitoneal injection of pentobarbital $(800 \mathrm{mg} / \mathrm{kg})[13,14]$ and brain tissues were removed according to different experimental requirements, or embedded in paraffin or ground into homogenate or other treatments. In each group, 6 brain tissues were ground into homogenate, 6 samples were used for staining, 3 were observed by transmission electron microscopy (TEM), and 6 were for detection of blood-brain barrier (BBB) and brain water content.

\subsection{Establishment of SAH in rats}

Rats were anesthetized with $60 \mathrm{mg} / \mathrm{kg}$ pentobarbital and $7.5 \mathrm{mg} / \mathrm{kg}$ xylazine intraperitoneally. Rats spontaneously breathed room air and were supplemented with oxygen to maintain $90 \%<\mathrm{O}_{2}<98 \%$ using 
pulse oximetry. The rearing temperature was maintained at $37^{\circ} \mathrm{C}$ using a rectal temperature sensor (Harvard Apparatus, Holliston, MA, USA). Surgical operations were carried out with aseptic techniques.

A sharpened 4- 0 nylon suture was inserted into the right external carotid artery and passed through the intracranial internal carotid artery; when confronted with resistance, the nylon suture was pushed $3 \mathrm{~mm}$ further to make the internal carotid artery wall perforated. Next, the suture was withdrawn, and the internal carotid artery was reperfused. Meanwhile, the filaments were introduced without arterial perforation in the sham controls. The severity of SAH was blindly evaluated as previously described [15]. The sub-arachnoid cistern was split into 6 segments, and each one was graded from 0 to 3. Grade 0 : no sub-arachnoid blood; grade 1: a small amount of sub-arachnoid blood; grade 2: moderate blood clot with recognizable arteries; and grade 3: blood clot in all arteries within the segment. The total score of each rat ranged from 0 to 18 .

\subsection{Isolation and culture of rat neurons}

After euthanasia with pentobarbital, the fetuses of 18-day pregnant rats were removed, and the hippocampus of the fetuses was stripped and detached with $0.25 \%$ trypsin. The hippocampus of fetal rats was added with neuron preculture media (modified Eagle's medium, 10\% fetal bovine serum, 1 $\mathrm{mmoL} / \mathrm{L}$ sodium pyruvate, $100 \mathrm{U}$ penicillin and streptomycin double antibody, $1 \times$ GlutaMAX) to stop the enzyme reaction. After repeated trituration, the hippocampus tissue was dispersed into single cells, counted and seeded into the culture dishes. After 3-5 hours, the media were changed into neuron media (neurobasal medium, $1 \times$ B27, $1 \times$ GlutaMAX, $100 \mathrm{U}$ penicillin and streptomycin), and the media were changed every 3 days.

The hippocampal neurons were allocated into blank group (normal cultured), FIN56 group (added with FIN56, a ferroptosis inducer), FIN56 + NC group (FIN56-induced neurons were transfected with adenovirus negative control), FIN56 + OTUB1 group (FIN56-induced neurons were transfected with adenovirus ubiquitin aldehyde binding 1 (OTUB1), a deubiquitylase), and FIN56 + TAK-243 group (FIN56-induced neurons were added with TAK-243, an ubiquitination inhibitor).

The recombinant adenovirus vector Ad-OTUB1 and the control Ad-NC were purchased from Hanbio Biotechnology Co., Ltd. China (Shanghai, China). FIN56 and TAK-243 were purchased from MedChemExpress Co., Ltd (Monmouth Junction, NJ, USA).

According to the instructions of Lipofectamine 2000 (Invitrogen, Carlsbad, CA, USA), Ad-OTUB1 and AdNC were transfected into neurons. FIN56 $(5 \mu \mathrm{m})$ [16] and TAK-243 $(1 \mathrm{~nm})$ [17] were applied to different groups of hippocampal neurons.

\subsection{Assessment of neurologic score, SAH grade and mortality}

Neurological scores were assessed at 24 and 72 hours in a blinded manner as previously reported [15]. An 18-point scoring system with 6 subsets (spontaneous activity, spontaneous movements of 4 limbs, 
forelimb outstretching, wire cage wall climb, trunk touch reaction and vibrissae touch response) was used. After that, the rats were sacrificed to quantify the severity of SAH [15]. Rats with score $<5$ points were excluded, and the mortality after the operation was recorded.

\subsection{Assessment of BBB permeability and brain water content}

BBB permeability was assessed using Evans blue extrapolation [18]. In each group, 6 rats were injected with $4 \%$ Evans blue dye $(2.5 \mathrm{~mL} / \mathrm{kg})$ into the left femoral vein under deep anesthesia 24 hours after operation to circulate for 60 minutes. The Evans blue dye was quantified using a spectrophotometer at $620 \mathrm{~nm}$ excitation wavelength, $680 \mathrm{~nm}$ emission wavelength and $10 \mathrm{~nm}$ bandwidth. To measure the water content in the brain, the brain was quickly divided into different parts under deep anesthesia, then weighed immediately (wet weight), and placed in the oven at $105^{\circ} \mathrm{C}$ (dry weight) for 72 hours. Brain water content $=$ (wet weight - dry weight) $/$ wet weight $\times 100 \%$.

\subsection{TEM observation}

The mitochondria in the brain of rats were observed by TEM. The rats were euthanized by pentobarbital at $24 \mathrm{~h}$ and $72 \mathrm{~h}$ after the operation respectively. The brain tissue of 3 rats in each group were removed quickly, first fixed at $4{ }^{\circ} \mathrm{C}$ for 2 hours in $2.5 \%$ glutaraldehyde in $0.1 \mathrm{M}$ phosphate buffer $(\mathrm{pH} 7.4)$, and then fixed for 1 hour in $1 \%$ osmium tetroxide on ice. Thereafter, tissues were embedded in Epon 12 after dehydration in a graded acetone series (up to 100\%), and sectioned using a Reichert ultramicrotome (Leica, Wetzlar, Germany) and stained with $3 \%$ uranyl acetate and lead citrate. Then, the sections were visualized under TEM (Technai G2 Spirit TWIN, FEI, USA) at $80 \mathrm{kV}$.

\subsection{Histological staining}

At 24 hours or 72 hours after the operation, the brain sections of rats were stained to observe the iron content and nerve injury.

After SAH for 24 hours or 72 hours, brain tissue sections were dewaxed. After staining with Nissl dyeing (Beyotime Biotechnology Co., Ltd, Shanghai, China), they were washed with distilled water, cleared in xylene, and sealed with neutral gum.

After SAH for 24 hours, the brain tissue sections were dewaxed and then put into Prussian blue dyeing

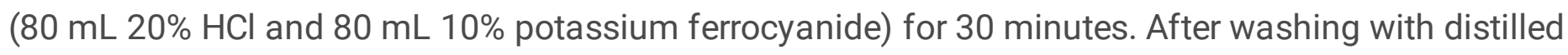
water, the sections were restained with eosin and observed under the microscope.

\subsection{Detection of ROS}

At 24 hours and 72 hours after the SAH operation, rats in each group were injected with $5 \mathrm{mmoL} / \mathrm{L}$ dihydroetorphine (DHE) working solution by tail vein. After 30 minutes, rats were killed and fresh brain tissues were taken for frozen sections. After dripping with anti-fluorescence quenching agent, the sections were blocked. At the same time, the nucleus was located using Hoechst staining. The neurons were 
incubated with DHE for 90 minutes at $37^{\circ} \mathrm{C}$ in the dark, and then washed with fresh media and observed under the fluorescence microscope.

\subsection{Detection of GSH and malondialdehyde (MDA) content}

The content of GSH and MDA in brain homogenate or neurons of SAH rats were determined in strict accordance with the instructions of the total glutathione test kit (S0052, Beyotime) and MDA test kit (S0131, Beyotime).

\subsection{Western blot analysis}

At 24 and 72 hours after the operation, the brain homogenates of rats in each group were taken, and the homogenates or neurons treated with different methods were lysed in cold radio-immunoprecipitation assay containing protease inhibitor cocktail (Sigma-Aldrich, Merck KGaA, Darmstadt, Germany) for 30 minutes. Then, the lysate was centrifuged at $4{ }^{\circ} \mathrm{C}$ for 20 minutes at $16000 \mathrm{~g}$, and the supernatant was collected. The protein concentration was quantified using a Pierce bicinchoninic acid protein assay kit (Beyotime). Equal amount of proteins (20 $\mu \mathrm{L} /$ well) were loaded for sodium dodecyl sulfatepolyacrylamide gel electrophoresis, and transferred to polyvinylidene fluoride membranes. Next, the membranes were blocked with 5\% skimmed milk powder for 1 hour, and probed with the primary antibodies and secondary antibody immunoglobulin G (IgG, 1:2000, ab205718, Abcam Inc., Cambridge, MA, USA). The gray values of target bands were analyzed with Image $J$ software, with $\beta$-actin as the internal reference. The used antibodies were p65 (1:1000, ab16502, Abcam), p-p65 (1:2000, ab86299, Abcam), FTH1 (1:1000, ab183781, Abcam), GPX4 (1:1000, ab125066, Abcam), $\beta$-actin (1:1000, ab8227, Abcam), SLC7A11 (1:1000, PA1-16893, Invitrogen), SLC3A2 (1:1000, MA5-31209, Invitrogen) and glutamate cysteine ligase catalytic (GCLC) (1:1000, ab190685, Abcam).

\subsection{Enzyme-linked immunosorbent assay (ELISA)}

The levels of interleukin (IL)-6, tumor necrosis factor-a (TNF- $a$ ), IL-1 $\beta$ and IL-18 in brain homogenates or neurons treated with different methods were detected in strict accordance with the instructions of the rat IL-6 quantikine ELISA kit (R6000B, R\&D Systems, Minneapolis, MN, USA), rat TNF-a quantikine ELISA kit (RTA00, R\&D Systems), rat IL-1 $\beta /$ IL-1F2 quantikine ELISA kit (RLB00, R\&D systems), and mouse IL-18 ELISA (7625, R\&D systems).

\subsection{Co-immunoprecipitation (Co-IP) assay}

Co-IP assay was performed according to the instructions provided by the supplier (Thermo Fisher, Waltham, MA, USA). The cells or tissues were dissolved in IP buffer supplemented with protease inhibitor. The supernatant was incubated with antibody against SLC3A2 or lgG antibody at $4{ }^{\circ} \mathrm{C}$ with protein $\mathrm{A} / \mathrm{G}$ agarose beads (Fast Flow, Beyotime) for 3 hours. The agarose beads were rinsed in cold phosphatebuffered saline, and then the binding proteins were eluted. The precipitated protein was measured by 
Western blot analysis and anti-ubiquitin. IgG was a control. Similarly, immunocoprecipitation of SLC7A11, GPx4 and ubiquitin C (UBC), SLC3A2 and OTUB1, GPX4 and OTUB1 was carried out in a similar way.

\subsection{Statistical analysis}

Statistical analysis was conducted by SPSS 21.0 (IBM Corp. Armonk, NY, USA). All the data were in normality distribution checked by the Kolmogorov-Smirnov test. Measurement data were expressed as mean \pm standard deviation. The $t$ test was used for comparisons between two groups. One-way or twoway analysis of variance (ANOVA) was used for comparisons among multiple groups, and Tukey's multiple comparisons test was used for post-hoc test. The $p$ value was obtained by a two-tailed test and $p<0.05$ indicated a significant difference.

\section{Results}

\subsection{SAH caused nerve defect, BBB damage and brain water content decrease in rats}

A total of 126 rats were enrolled in this study, and SAH was induced in 78 rats. Due to the severity of SAH, 9 rats died immediately during or after the operation, and 11 rats were excluded from the study due to the low SAH grade after the operation, and the rest 48 rats were used as sham controls. Postoperatively, the rats showed symptoms of $\mathrm{SAH}$, including drowsiness, weakness and moaning, indicating severe headache in the rats. Some SAH rats showed ischemia-like hemiplegia and hemiparalysis, occasionally with drooping eyelids on the same side. In addition to the 20 rats that died immediately after operation and had insufficient SAH level, another 10 rats died within 24 hours after operation in the remaining 58 rats (Table 1). No death was observed in the sham group. At 24 hours after the operation, different degrees of bleeding were observed in the subarachnoid space of rats and SAH was scored, which proved that our model was successfully constructed (Fig. 1A/B). SAH significantly induced postoperative neurological dysfunction. Compared with sham-operated rats, the SAH rats showed significantly decreased neurological scores at 24 and 72 hours ( $p<0.05$; Fig. 1C). Evans blue dye measured the permeability of BBB, and found that SAH caused significant BBB damage in cerebral hemisphere, cerebellum (CB) and brain stem (BS) 72 hours after operation (Fig. 1D). After SAH operation, especially at 72 hours, the diffuse brain swelling was caused. Compared with sham-operated rats, SAH rats showed significantly decreased brain water content of left hemisphere $(\mathrm{LH})$, right hemisphere $(\mathrm{RH})$ and $\mathrm{CB}$ at 72 hours (Fig. 1E). 
Table 1

Mortality rate of rats in each group

\begin{tabular}{|llll|}
\hline Group & Death & Total rats & Mortality rate (\%) \\
\hline Sham & 0 & 48 & 0 \\
\hline SAH & 10 & 58 & 17.24 \\
\hline
\end{tabular}

\subsection{SAH rats showed ferroptosis and downregulated GPX4 in hippocampal neurons}

After Nissl staining of SAH rat brain tissues, we found a significant decrease in hippocampal neurons (Fig. 2A). Then we used DHE fluorescence to label ROS in SAH rat hippocampus, and found that compared with sham-operated rats, SAH rats exhibited increased red fluorescence in the brain, which was accumulated over time (Fig. 2B), indicating that SAH caused serious brain damage and ROS accumulation. Ferroptosis is featured with GSH depletion, disrupted GPX4 redox defense, mitochondrial damage, inflammation and detrimental lipid ROS formation $[8,19]$. Thus, we suspected that the excessive ROS in the hippocampus of SAH rats may be caused by neuronal ferroptosis. So we detected the iron deposition in the brain of SAH rats. The iron deposition in the brain of SAH rats increased gradually with time, and the mitochondrion membrane in the hippocampal neurons of SAH rats broke and bubbled (Fig. 2A). Western blot analysis showed that GPX4 and FTH1 (ferroptosis related protein) in the brain of SAH rats were significantly downregulated (both $p<0.05$; Fig. $2 \mathrm{C}$ ). After that, $\mathrm{GSH}$ and MDA levels in the brain of SAH rats were detected and it was found that GSH was exhausted, MDA level was significantly increased (both $p<0.05$; Fig. 2D), and a large number of inflammatory factors (IL-6, TNF-a, IL-1 $\beta$, and IL18) were produced (Fig. 2E), which indicated that SAH caused neuron ferroptosis in the hippocampus of SAH rats.

\subsection{System $\mathrm{Xc}^{-}$function was lost in the brain of SAH rats}

After observing the ferroptosis in hippocampal neurons in SAH rats, we wanted to know why and how the ferroptosis happened. So we looked up the ferroptosis related signaling pathway (Fig. 3A) from KEGG (https://www.kegg.jp). In the upstream of GPX4 (a key protein to ferroptosis), we focused on System Xc' an amino acid transporter, which consists of two functional subunits, SLC3A2 (the solute carrier family 3, member 2) and SLC7A11 (solid carrier family 7, member 11). System $\mathrm{Xc}^{-}$transports cystine into cells through 1:1 reverse transport of glutamic acid and participates in the synthesis of GSH; inhibition of System $\mathrm{Xc}^{-}$leads to neuronal cell death [20]. We detected SLC3A2 and SLC7A11 expression in the brain of SAH rats, and discovered that compared with the sham-operated rats, the SAH rats showed significantly reduced SLC3A2 and SLC7A11 levels in the brain, and the expression of GCLC was also significantly reduced (all $p<0.05$; Fig. 3B/C), indicating the loss of System $\mathrm{Xc}^{-}$function in the brain of 
SAH rats. Overall, the loss of System $\mathrm{Xc}^{-}$function blocked the synthesis of GSH, inhibited GPX4 function, and caused oxidative damage, ROS accumulation and ferroptosis of hippocampal neurons.

\subsection{System $\mathrm{Xc}^{-}$and GPX4 interacted with ubiquitination- related proteins}

In the SAH rats, we observed the loss of System $\mathrm{Xc}^{-}$function and ferroptosis of hippocampal neurons. To further explore the molecular mechanism of System $\mathrm{Xc}^{-}$in the ferroptosis of hippocampal neurons in SAH rats, we predicted the interactions between GPX4 and System $\mathrm{Xc}^{-}$(SLC3A2 and SLC7A11) online in the UNIHI database (http://193.136.227.168/unihi/pages/unihisearch.jsf) (Fig. 4A). We found they were interacted with UBC and OTUB1. Moreover, it has been reported that OTUB1 mediates ferroptosis by stabilizing SLC7A11 [21]. Therefore, we speculated that System $\mathrm{Xc}^{-}$and GPX4 regulated the synthesis and consumption of GSH in neurons through ubiquitination and deubiquitination, so as to maintain the dynamic balance of GSH level. But SAH disrupted the balance and led to the decrease of GSH synthesis and increase of GSH consumption in cells. The ubiquitination of SLC3A2, SLC7A11 and GPX4 in the brain of SAH rats was detected by Co-IP assay. It was found that SLC3A2, SLC7A11 and GPX4 were all ubiquitinated, and the expression of OTUB1 was significantly decreased (all $p<0.05 ; \mathrm{Fig}$. 4B/C). Then we verified the interaction between SLC3A2 and OTUB1, GPX4 and OTUB1 in the isolated and cultured hippocampal neurons of rats (Fig. 4D).

\subsection{Overexpression of OTUB1 alleviated ferroptosis in neurons after SAH}

To further verify the mechanism of ferroptosis, we applied FIN56 (a kind of ferroptosis inducer) [16] to cultured hippocampal neurons to induce ferroptosis. The ubiquitination degradation of SLC3A2, SLC7A11 and GPX4 and the significant downregulation of FTH1 and OTUB1 were observed in the ferroptotic hippocampal neurons (all $p<0.05$; Fig. 5B-C). GSH depletion (Fig. 5D) and the production of a large number of ROS and inflammatory factors (Fig. 5E-F) were also observed. After overexpression of OTUB1 in hippocampal neurons (Fig. 5A), we found that the ubiquitination of SLC3A2, SLC7A11 and GPX4 was decreased, FTH1 expression and GSH content were increased, and the level of ROS and inflammatory factors were decreased (Fig. 5B-F, all $p<0.01$ ). It can be concluded that the ferroptosis of hippocampal neurons is related to the imbalance of ubiquitination and deubiquitination of System $\mathrm{Xc}^{-}$and GPX4.

\subsection{Inhibition of System $\mathrm{Xc}^{-}$and GPx4 ubiquitination in neurons reduced ferroptosis-induced injury}

Next, we used the ubiquitination inhibitor (TAK-243) to reduce the ubiquitination of SLC3A2, SLC7A11 and GPX4 in FIN56-induced ferroptotic neurons (Fig. 6A). It was found that after the ubiquitination of SLC3A2, SLC7A11 and GPX4 was decreased, the GSH and MDA contents in neurons were increased significantly 
(all $p<0.05$; Fig. 6B), which further confirmed the role of System $\mathrm{Xc}^{-}$and GPX4 ubiquitination in neuron ferroptosis.

\section{Discussion}

SAH accounts for approximately $5-10 \%$ of all strokes, and the mortality rate is about $30-50 \%$ and $40-$ $50 \%$ of patients recovering from it experience severe neurological deficits [2]. Ferroptosis underlies spontaneous human diseases, such as major neurodegeneration and neuroinflammation, causing an excessive cell death and activation of lipid peroxidation [10]. Moreover, most of proteins (such as a subunit of SLC7A11, GPX4, and GCLC) that interact in the pathway of ferroptosis are controlled by antioxidant response elements [12]. In this study, we unveiled that SAH induced the ubiquitination of System $\mathrm{Xc}^{-}$and GPX4, resulting in GSH depletion, increased lipid oxidative damage and promoted ferroptosis in hippocampal neurons, and inhibition of System $\mathrm{Xc}^{-}$and GPX4 ubiquitination relieved ferroptosis-induced neuron damage in SAH rats (Fig. 7).

Neuron death induced by ROS is a vital mechanism of early brain injury after SAH [22]. The histological staining in the present study verified that SAH caused a significant decrease in hippocampal neurons and serious brain damage and ROS accumulation. Oxidative stress and antioxidant are key modulators of lipid peroxidation that trigger ferroptosis [12]. Ferroptotic cells display smaller mitochondria with ruptured outer membrane [6]. The iron deposition in the brain of SAH rats increased gradually with time, and the mitochondrion membrane in hippocampal neurons broke and bubbled. Ferroptosis is driven by the loss of GPX4 activities and ROS accumulation [23]. GPX4 and FTH1 in the brain of SAH rats were significantly downregulated, GSH was exhausted, MDA level was increased, and inflammatory cytokines were produced. Inflammation in the brain vessels and the subarachnoid space is solely accounted to occur with or aggravate cerebral vasospasm following SAH [24]. GPX4 is the only enzyme that can eliminates and directly detoxify lipid hydroperoxides in biological membranes [25]. Ferroptosis induces GSH depletion, disrupted GPX4 function, mitochondrial damage, inflammation and ROS production [8]. Taken together, SAH rats showed ferroptosis and downregulated GPX4 in hippocampal neurons.

To further identify how ferroptosis happened, we searched the KEGG for ferroptosis-related pathway, and focused on System $\mathrm{Xc}^{-}$. Glutamate-mediated System $\mathrm{Xc}^{-}$inhibition leads to neuronal cell death, GSH depletion and oxidative stress [20]. In this study, SAH rats showed significantly reduced expression of SLC3A2, SLC7A11, and GCLC in the brain. GSH is the most decreased cellular metabolite during ferroptosis, and GSH depletion causes loss of cellular antioxidant capacity and inhibition of GPXs [6, 26]. In brief, the loss of System $\mathrm{Xc}^{-}$function blocked GSH synthesis and GPX4 function, and caused oxidative damage, ROS accumulation and ferroptosis of hippocampal neurons. The biological significance of ferroptosis is expanding rapidly thanks to the discovery of GPX4 and System $\mathrm{Xc}^{-}$regulation in ferroptosis [23]. To further explore the molecular mechanism of System $\mathrm{Xc}^{-}$in the ferroptosis, we found GPX4 and System $\mathrm{XC}^{-}$(SLC3A2 and SLC7A11) were interacted with ubiquitin $\mathrm{C}$ and OTUB1. SLC3A2, SLC7A11 and GPX4 were all ubiquitinated, and OTUB1 expression was significantly decreased. The deubiquitylase 
OTUB1 mediates ferroptosis by directly interacting with SLC7A11 and regulating SLC7A11 stability in a p53-independent manner [21]. Ubiquitination is vital to any eukaryotic cell under oxidative stress, where ubiquitination levels are drastically increased, and elevated levels of ubiquitin-proteins are observed in the brain after focal and global cerebral ischemia [27]. In other words, SAH disrupted the balance of ubiquitination and deubiquitination of System $\mathrm{Xc}^{-}$and GPX4, and led to the decrease of GSH synthesis and increase of GSH consumption in hippocampal neurons.

FIN56 has been identified to cause loss of GPX4 activity and overwhelming lipid peroxidation, resulting ultimately in ferroptosis [28]. So we used FIN56 to induce ferroptosis in hippocampal neurons. The results revealed that FIN56 treatment contributed to ubiquitination degradation of SLC3A2, SLC7A11 and GPX4, the significant downregulation of FTH1 and OTUB1, GSH depletion, and the production of ROS and inflammatory factors. Moreover, after overexpression of OTUB1 (a deubiquitylase) or treatment with TAK243 (an ubiquitination inhibitor) in hippocampal neurons, the ubiquitination of SLC3A2, SLC7A11 and GPX4 was decreased, FTH1 expression and GSH content were increased, and the levels of ROS and inflammatory factors were decreased. These further confirmed the role of System $\mathrm{Xc}^{-}$and GPX4 ubiquitination in neuron ferroptosis. We concluded that the ferroptosis of hippocampal neurons is related to the imbalance of ubiquitination and deubiquitination of System $\mathrm{Xc}^{-}$and GPX4. Inhibition of System Xc- and GPX4 ubiquitination in neurons reduced ferroptosis-induced injury.

\section{Conclusion}

In Conclusions, the importance of ferroptosis in development is evident clinically in acute kidney injury, Huntington diseases, periventricular leukomalacia, and cancers [23]. But the investigation of ferroptosis in hippocampal neurons of SAH rats is less touched. Our study highlighted that the loss of System $\mathrm{Xc}^{-}$ function after SAH blocked GSH synthesis and GPX4 function, and caused oxidative damage and ROS accumulation, thus leading to ferroptosis of hippocampal neurons. We provided a novel mechanism of ferroptosis in SAH. However, there are several limitations in this study. First, the regulatory mechanism of SAH is very complicated, and we just explored the involvement of ferroptosis in the process. More studies in the future are required to identify the comprehensive mechanism of SAH. Second, we only studied the damage conditions within 72 hours after the SAH operations in the rat SAH model. The mechanism of brain injury after 72 hours needs to be studied. Nonetheless, the present results implied that the presence of ferroptosis after $\mathrm{SAH}$, and may provide new therapeutic clues in patients with $\mathrm{SAH}$.

\section{Abbreviations}

GPX4: Glutathione peroxidase 4; SAH: subarachnoid hemorrhage ; ROS : reactive oxygen species ; GSH : glutathione; TEM : transmission electron microscopy; BBB : blood-brain barrier; DHE : dihydroetorphine; MDA : malondialdehyde; IgG : immunoglobulin G; GCLC : glutamate cysteine ligase catalytic; ELISA : Enzyme-linked immunosorbent assay; IL : interleukin; TNF-a : tumor necrosis factor-a; Co-IP : Co- 
immunoprecipitation; UBC : ubiquitin C; ANOVA : analysis of variance; CB : cerebellum; BS : brain stem; LH : left hemisphere; RH : right hemisphere; OTUB1: ubiquitin aldehyde binding 1.

\section{Declarations}

\section{Ethics Approval and consent to participate}

This study was performed with the approval of the Clinical Ethical Committee of Renmin Hospital of Wuhan University. All procedures were strictly conducted in accordance with the code of ethics. Significant efforts were made to minimize the animals used and their suffering.

\section{Competing Interests}

The authors declare that they have no conflict of interests.

\section{Availability of Data and Materials}

The data that support the findings of this study are available from the corresponding author upon reasonable request.

\section{Consent for Publication}

Not applicable.

\section{Funding}

This study was supported by Nanjing Military Area Research Fund Major Project(NO.15DX003),Youth Project of Wuxi Natural Science Foundation (NO. Q201611) and Wuxi Science and Technology Development Fund Project (NO.WX18IIAN041).

\section{Authors' Contributions}

$\mathrm{JHC}$ is the guarantor of integrity of the entire study and contributed to the concepts and design of this study; CLZ, YHW and QXC contributed to the definition of intellectual content and literature research of this study; ZHL and MCLcontributed to the experimental studies and the data acquisition and analysis; SY,SMH and QT took charge of the manuscript preparation and review. All authors read and approved the final manuscript. 


\section{Acknowledgments}

Not applicable.

\section{References}

1. Ogunlaja Ol, Cowan R: Subarachnoid Hemorrhage and Headache. Curr Pain Headache Rep 2019, 23:44.

2. Richard SA: Elucidating the novel biomarker and therapeutic potentials of High-mobility group box 1 in Subarachnoid hemorrhage: A review. AIMS Neurosci 2019, 6:316-332.

3. Long B, Koyfman A, Runyon MS: Subarachnoid Hemorrhage: Updates in Diagnosis and Management. Emerg Med Clin North Am 2017, 35:803-824.

4. Li B, Luo C, Tang W, Chen Z, Li Q, Hu B, Lin J, Zhu G, Zhang JH, Feng H: Role of HCN channels in neuronal hyperexcitability after subarachnoid hemorrhage in rats. J Neurosci 2012, 32:3164-3175.

5. Nirmala JG, Lopus M: Cell death mechanisms in eukaryotes. Cell Biol Toxicol 2020, 36:145-164.

6. Yang WS, SriRamaratnam R, Welsch ME, Shimada K, Skouta R, Viswanathan VS, Cheah JH, Clemons PA, Shamji AF, Clish CB, et al: Regulation of ferroptotic cancer cell death by GPX4. Cell 2014, 156:317-331.

7. Dixon SJ, Lemberg KM, Lamprecht MR, Skouta R, Zaitsev EM, Gleason CE, Patel DN, Bauer AJ, Cantley AM, Yang WS, et al: Ferroptosis: an iron-dependent form of nonapoptotic cell death. Cell 2012, 149:1060-1072.

8. Jelinek A, Heyder L, Daude M, Plessner M, Krippner S, Grosse R, Diederich WE, Culmsee C: Mitochondrial rescue prevents glutathione peroxidase-dependent ferroptosis. Free Radic Biol Med 2018, 117:45-57.

9. Echigo R, Shimohata N, Karatsu K, Yano F, Kayasuga-Kariya Y, Fujisawa A, Ohto T, Kita Y, Nakamura $M$, Suzuki S, et al: Trehalose treatment suppresses inflammation, oxidative stress, and vasospasm induced by experimental subarachnoid hemorrhage. J Trans/ Med 2012, 10:80.

10. Maiorino M, Conrad M, Ursini F: GPx4, Lipid Peroxidation, and Cell Death: Discoveries, Rediscoveries, and Open Issues. Antioxid Redox Signa/ 2018, 29:61-74.

11. Chen L, Hambright WS, Na R, Ran Q: Ablation of the Ferroptosis Inhibitor Glutathione Peroxidase 4 in Neurons Results in Rapid Motor Neuron Degeneration and Paralysis. J Biol Chem 2015, 290:2809728106.

12. Kajarabille N, Latunde-Dada GO: Programmed Cell-Death by Ferroptosis: Antioxidants as Mitigators. Int J Mol Sci 2019, 20.

13. Kopaladze RA: [Methods for the euthanasia of experimental animals-the ethics, esthetics and personnel safety]. Usp Fiziol Nauk 2000, 31:79-90.

14. Zatroch KK, Knight CG, Reimer JN, Pang DS: Refinement of intraperitoneal injection of sodium pentobarbital for euthanasia in laboratory rats (Rattus norvegicus). BMC Vet Res 2017, 13:60. 
15. Sugawara T, Ayer R, Jadhav V, Zhang JH: A new grading system evaluating bleeding scale in filament perforation subarachnoid hemorrhage rat model. J Neurosci Methods 2008, 167:327-334.

16. Shimada K, Skouta R, Kaplan A, Yang WS, Hayano M, Dixon SJ, Brown LM, Valenzuela CA, Wolpaw AJ, Stockwell BR: Global survey of cell death mechanisms reveals metabolic regulation of ferroptosis. Nat Chem Biol 2016, 12:497-503.

17. Hyer ML, Milhollen MA, Ciavarri J, Fleming P, Traore T, Sappal D, Huck J, Shi J, Gavin J, Brownell J, et al: A small-molecule inhibitor of the ubiquitin activating enzyme for cancer treatment. Nat Med 2018, 24:186-193.

18. Tsubokawa T, Solaroglu I, Yatsushige H, Cahill J, Yata K, Zhang JH: Cathepsin and calpain inhibitor E64d attenuates matrix metalloproteinase-9 activity after focal cerebral ischemia in rats. Stroke 2006, 37:1888-1894.

19. Li X, Wang TX, Huang X, Li Y, Sun T, Zang S, Guan KL, Xiong Y, Liu J, Yuan HX: Targeting ferroptosis alleviates methionine-choline deficient (MCD)-diet induced NASH by suppressing liver lipotoxicity. Liver Int 2020.

20. Lewerenz J, Hewett SJ, Huang Y, Lambros M, Gout PW, Kalivas PW, Massie A, Smolders I, Methner A, Pergande $\mathrm{M}$, et al: The cystine/glutamate antiporter system $\mathrm{x}(\mathrm{c})(-)$ in health and disease: from molecular mechanisms to novel therapeutic opportunities. Antioxid Redox Signal 2013, 18:522-555.

21. Liu T, Jiang L, Tavana O, Gu W: The Deubiquitylase OTUB1 Mediates Ferroptosis via Stabilization of SLC7A11. Cancer Res 2019, 79:1913-1924.

22. Yang Y, Chen S, Zhang JM: The Updated Role of Oxidative Stress in Subarachnoid Hemorrhage. Curr Drug Deliv 2017, 14:832-842.

23. Yang WS, Stockwell BR: Ferroptosis: Death by Lipid Peroxidation. Trends Cell Biol 2016, 26:165-176.

24. Schneider UC, Xu R, Vajkoczy P: Inflammatory Events Following Subarachnoid Hemorrhage (SAH). Curr Neuropharmacol 2018, 16:1385-1395.

25. Grim JM, Hyndman KA, Kriska T, Girotti AW, Crockett EL: Relationship between oxidizable fatty acid content and level of antioxidant glutathione peroxidases in marine fish. $J$ Exp Bio/ 2011, 214:37513759.

26. Brigelius-Flohe R, Maiorino M: Glutathione peroxidases. Biochim Biophys Acta 2013, 1830:32893303.

27. Hochrainer K: Protein Modifications with Ubiquitin as Response to Cerebral Ischemia-Reperfusion Injury. Trans/ Stroke Res 2018, 9:157-173.

28. Conrad M, Kagan VE, Bayir H, Pagnussat GC, Head B, Traber MG, Stockwell BR: Regulation of lipid peroxidation and ferroptosis in diverse species. Genes Dev 2018, 32:602-619.

\section{Figures}


A

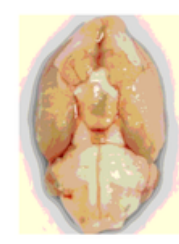

Sham

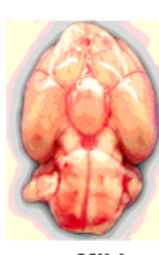

Mild

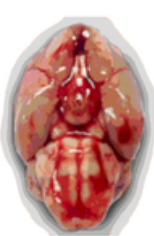

Moderate

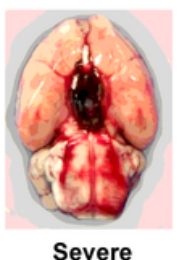

D

B

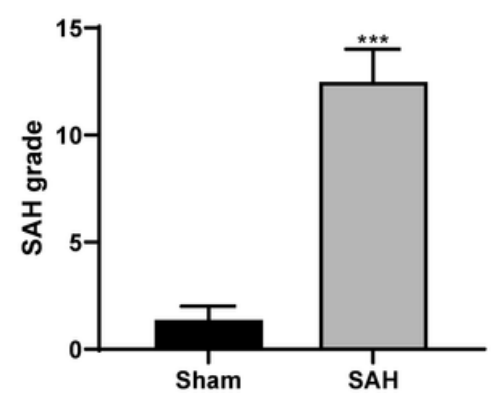

$\mathbf{E}$
C

- Sham $\square \mathrm{SAH}$

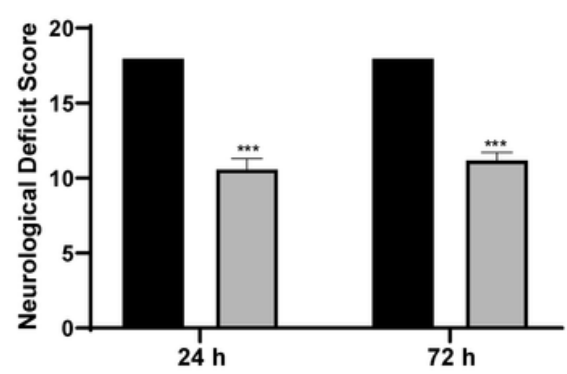

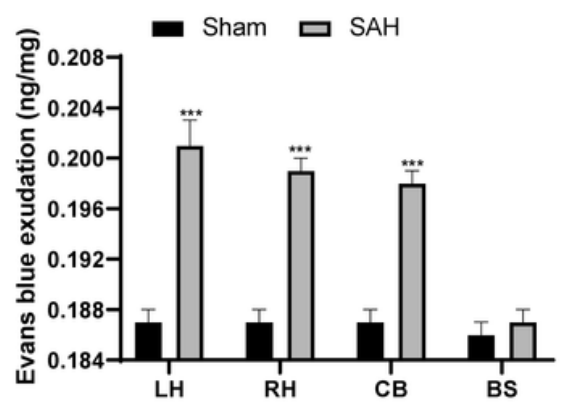

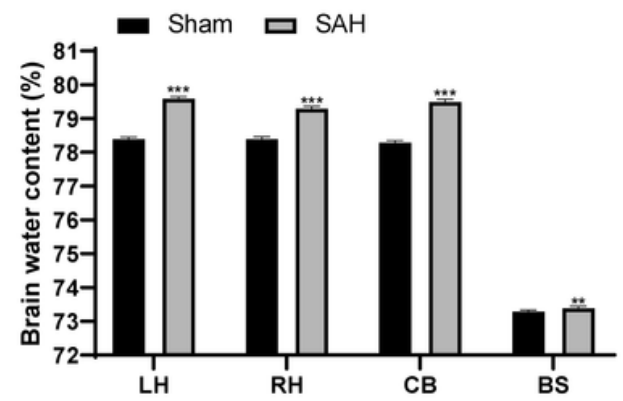

Figure 1

SAH caused nerve defect, BBB damage and brain water content decrease in rats. A-B, the degree of SAH in rats 24 hours after operation and SAH score $(n=48) ; C$, neurological deficit score of rats after the SAH operation at 24 hours and 72 hours. An 18-point scoring system consisting of 6 subsets (spontaneous activity, spontaneous movements of 4 limbs, forelimb outstretching, wire cage wall climb, trunk touch reaction and vibrissae touch response) was used $(n=6) ; D$, Evans blue staining was used to observe the permeability of BBB $(n=6)$; E: assessment of brain water content $(n=6)$. $\star \star \star ~ p<0.001$, compared with the sham group. The data in panel $\mathrm{B}$ were analyzed using the $t$ test, and the data in panels $\mathrm{C}, \mathrm{D}$ and $\mathrm{E}$ were analyzed using two-way ANOVA, followed by Tukey's multiple comparisons test. 

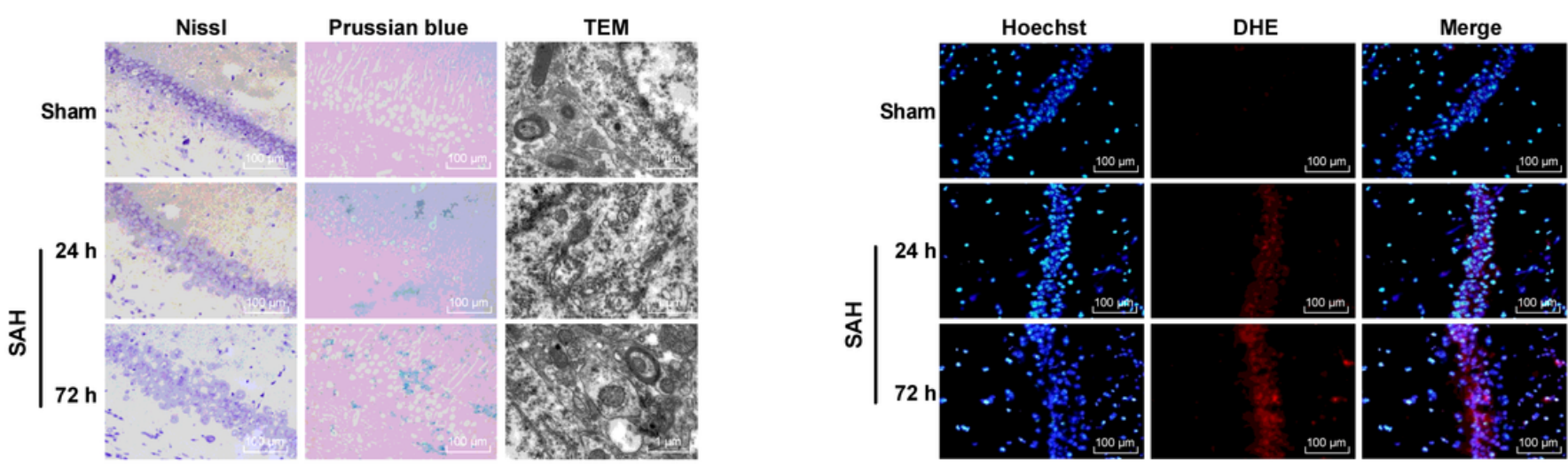

C

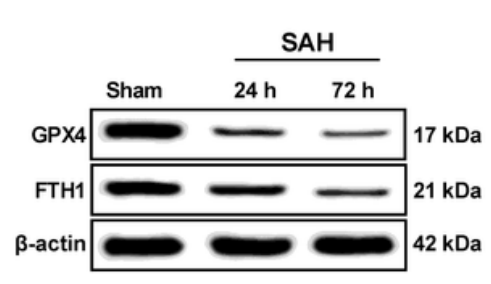

$\mathbf{E}$

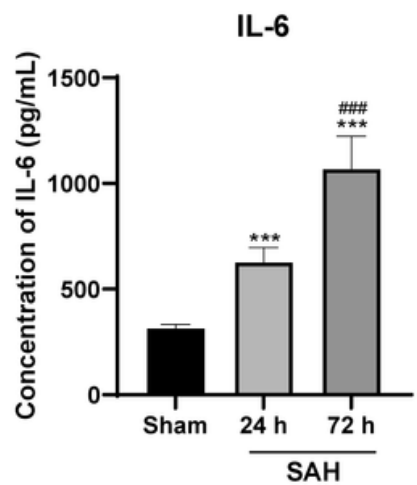

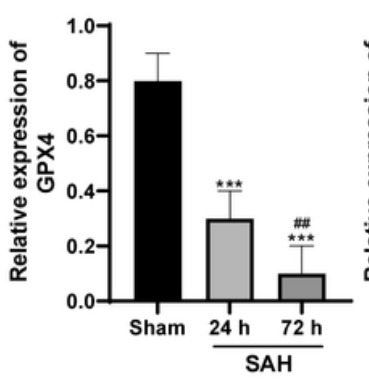

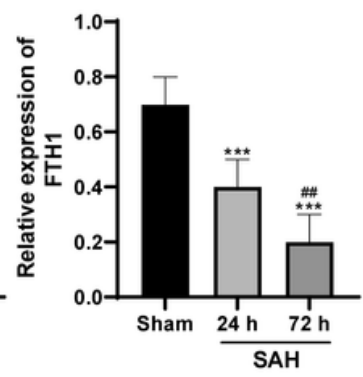

D
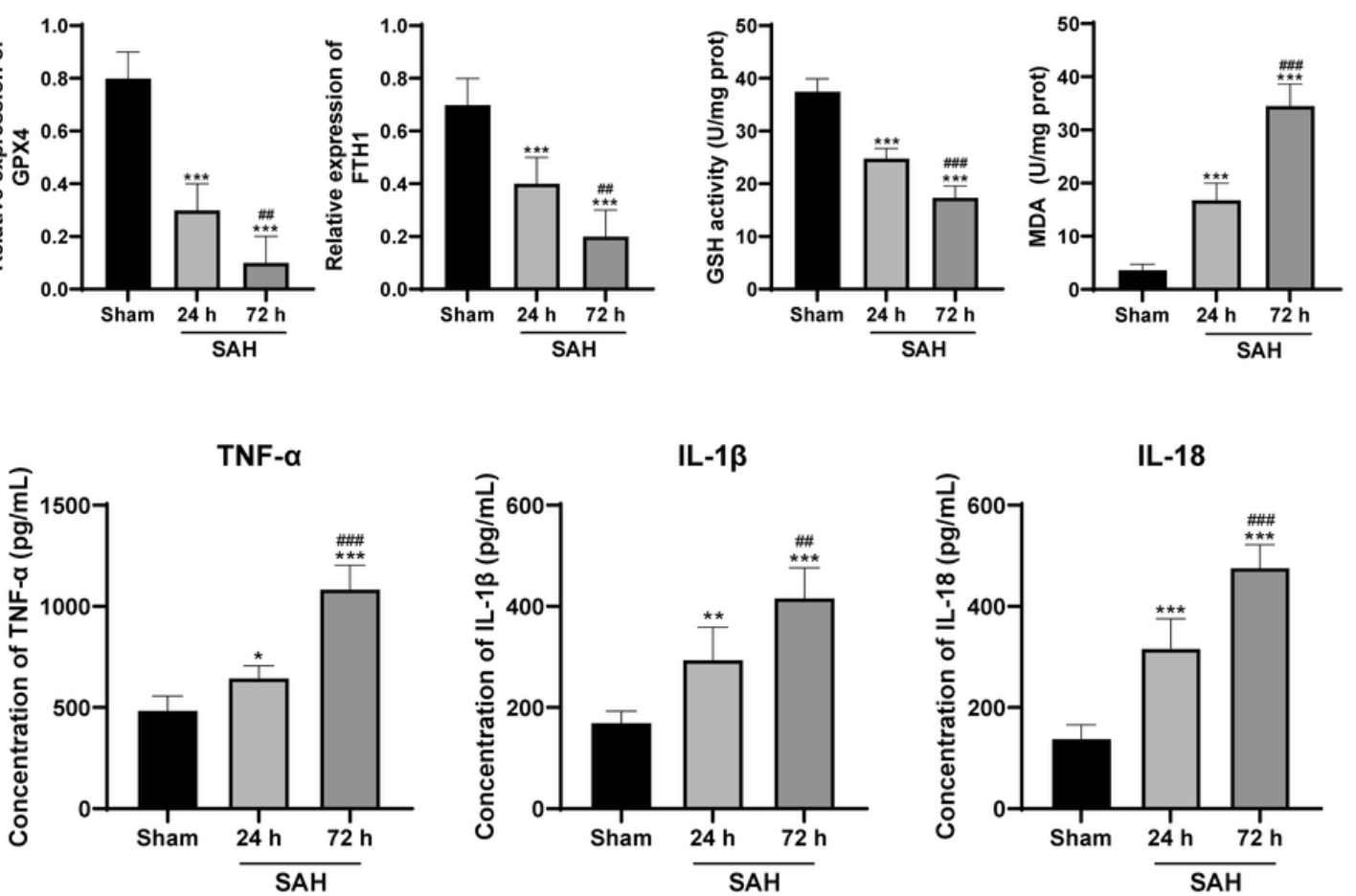

Figure 2

SAH caused nerve defect, BBB damage and brain water content decrease in rats. A-B, the degree of SAH in rats 24 hours after operation and SAH score $(n=48)$; C, neurological deficit score of rats after the SAH operation at 24 hours and 72 hours. An 18-point scoring system consisting of 6 subsets (spontaneous activity, spontaneous movements of 4 limbs, forelimb outstretching, wire cage wall climb, trunk touch reaction and vibrissae touch response) was used $(n=6) ; D$, Evans blue staining was used to observe the permeability of BBB $(n=6)$; E: assessment of brain water content $(n=6)$. ${ }^{\star \star *} p<0.001$, compared with the sham group. The data in panel $B$ were analyzed using the $t$ test, and the data in panels $C, D$ and $E$ were analyzed using two-way ANOVA, followed by Tukey's multiple comparisons test. 

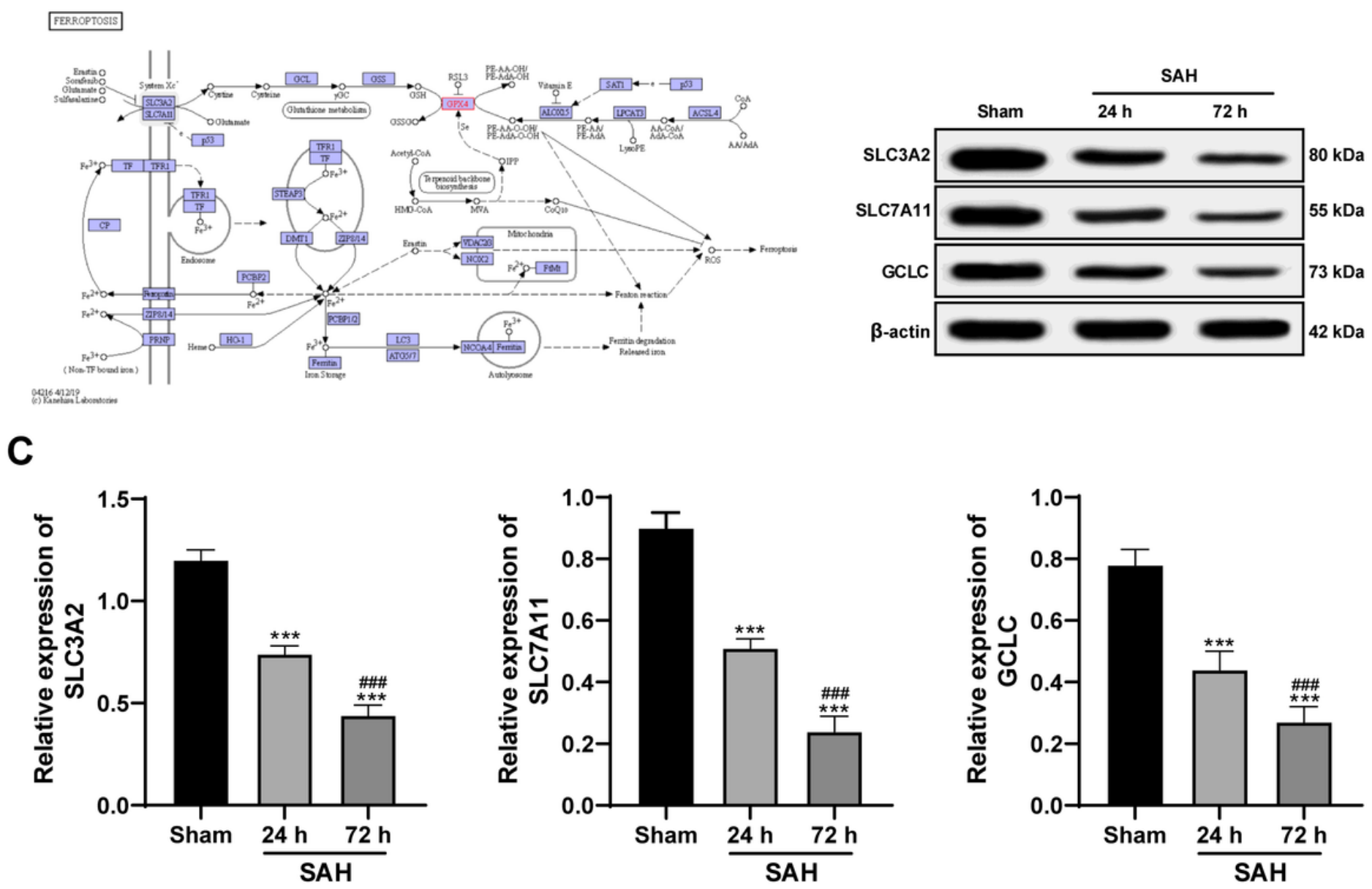

Figure 3

System Xc- function was lost in the brain of SAH rats. A, the ferroptosis related signaling pathway from KEGG database; B-C, levels of SLC3A2, SLC7A11 and GCLC in the brain of SAH rats were measured by Western blot analysis. Compared with the sham group, ${ }^{\star \star \star} p<0.001$; compared with the 24 hours, \#\#\# $p$ $<0.001$. The data were analyzed using one-way ANOVA, followed by Tukey's multiple comparisons test. $n=6$. 
A

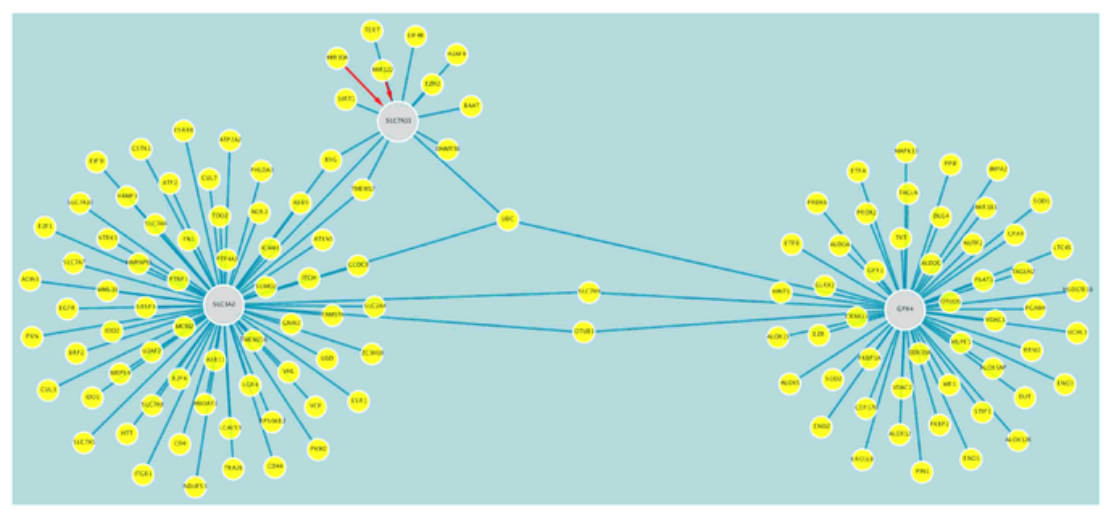

B
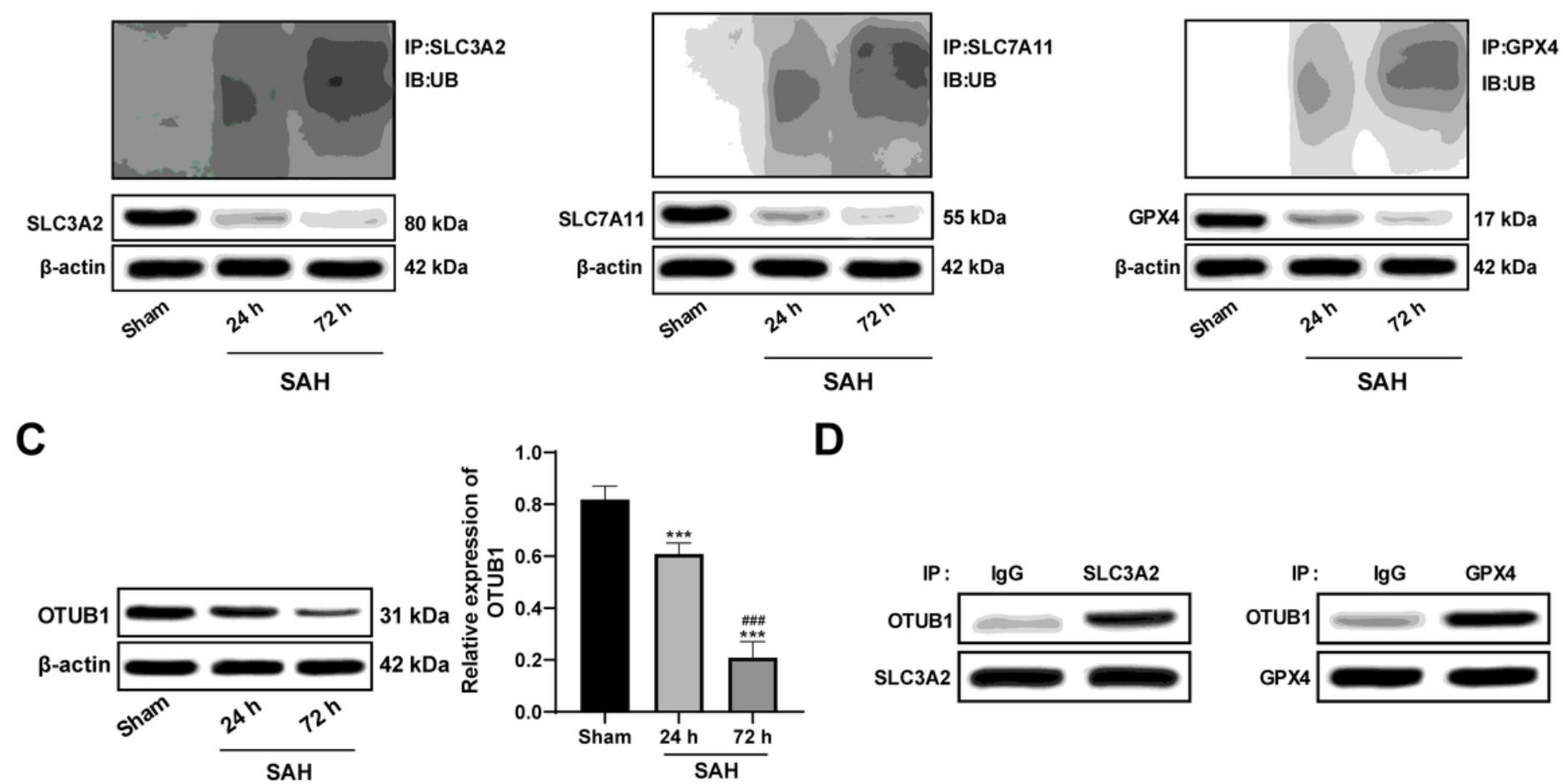

Figure 4

System Xc- and GPX4 interacted with ubiquitination-related proteins. A, the interactions between GPX4 and System Xc- (SLC3A2 and SLC7A11) were predicted online in the UNIHI database (http://193.136.227.168/unihi/pages/unihisearch.jsf); B, ubiquitination of SLC3A2, SLC7A11 and GPX4 in the brain of SAH rats was detected by Co-IP assay and Western blot analysis; $\mathrm{C}$, the expression of OTUB1 in the brain of SAH rats was detected by western blot analysis; $D$, the interactions between SLC3A2 and OTUB1, GPX4 and OTUB1 were verified by Co-IP assay. Compared with the sham group, *** $p<0.001$; compared with the 24 hours, \#\#\# $p<0.001$. The data were analyzed using one-way ANOVA, followed by Tukey's multiple comparisons test. $n=6$. 

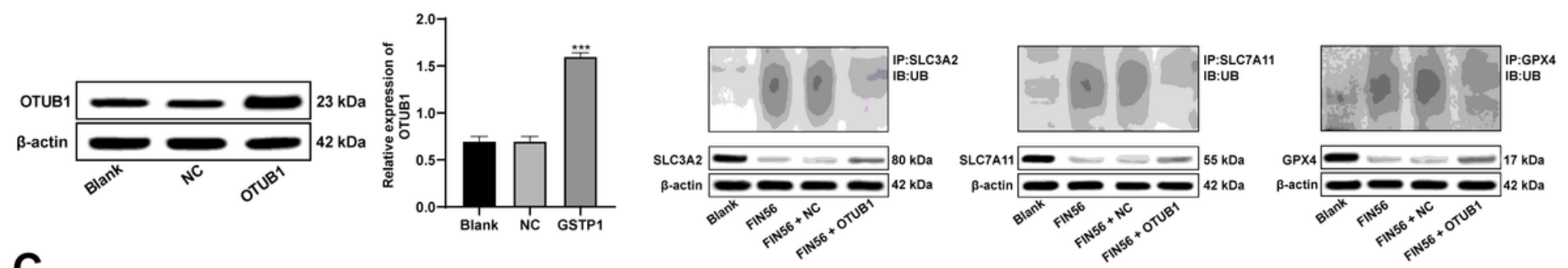

C

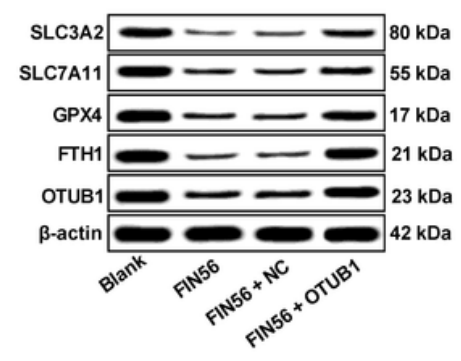

D

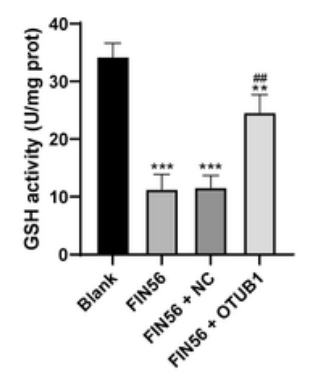

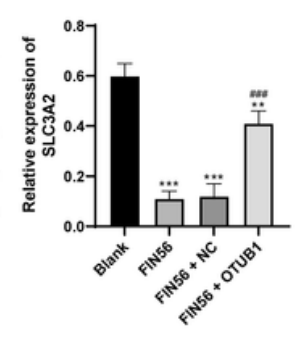

$\mathbf{E}$
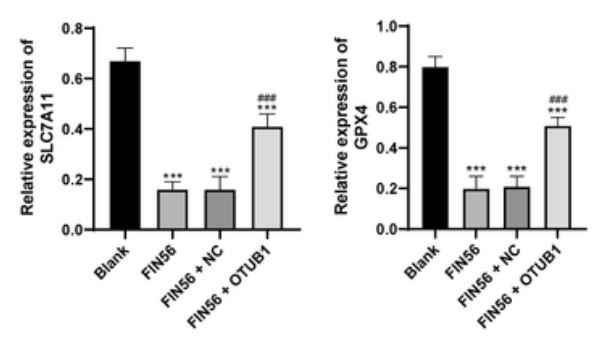
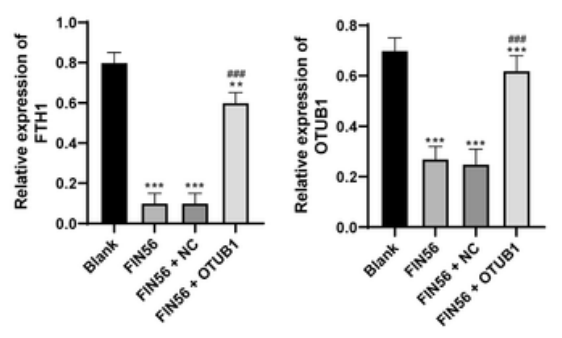

$\mathbf{F}$
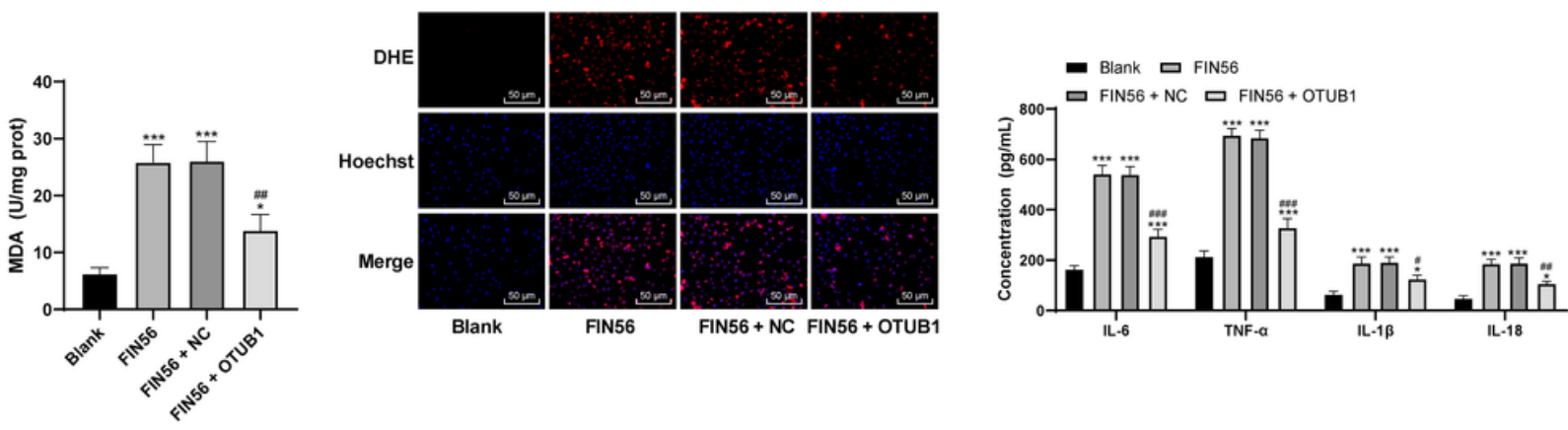

Figure 5

Ferroptosis of hippocampal neurons is related to the imbalance of ubiquitination and deubiquitination of System Xc- and GPX4. A, the expression of OTUB1 in hippocampal neurons was detected by Western blot analysis; B, ubiquitination of SLC3A2, SLC7A11 and GPX4 in the brain of SAH rats was detected by Co-IP assay and western blot analysis; C, expression of SLC3A2, SLC7A11, GPX4, FTH1 and OTUB1 was detected by Western blot analysis; D, GSH and MDA levels in the brain of SAH rats were detected by colorimetry; E, ROS level was observed by DHE probes; F, levels of inflammatory cytokines (IL-6, TNF-a, IL$1 \beta$ and IL-18) in the brain of SAH rats were measured by ELISA kits. Compared with the blank group, ** $p$ $<0.01$, $* * * p<0.001$; compared with the FIN56 group, \#\# $p<0.01$, \#\#\#p<0.001. The data in panels $A, C$ and $\mathrm{D}$ were analyzed using one-way ANOVA, and data in panel $\mathrm{F}$ were analyzed using two-way ANOVA, followed by Tukey's multiple comparisons test. Replicates $=3$. 
A
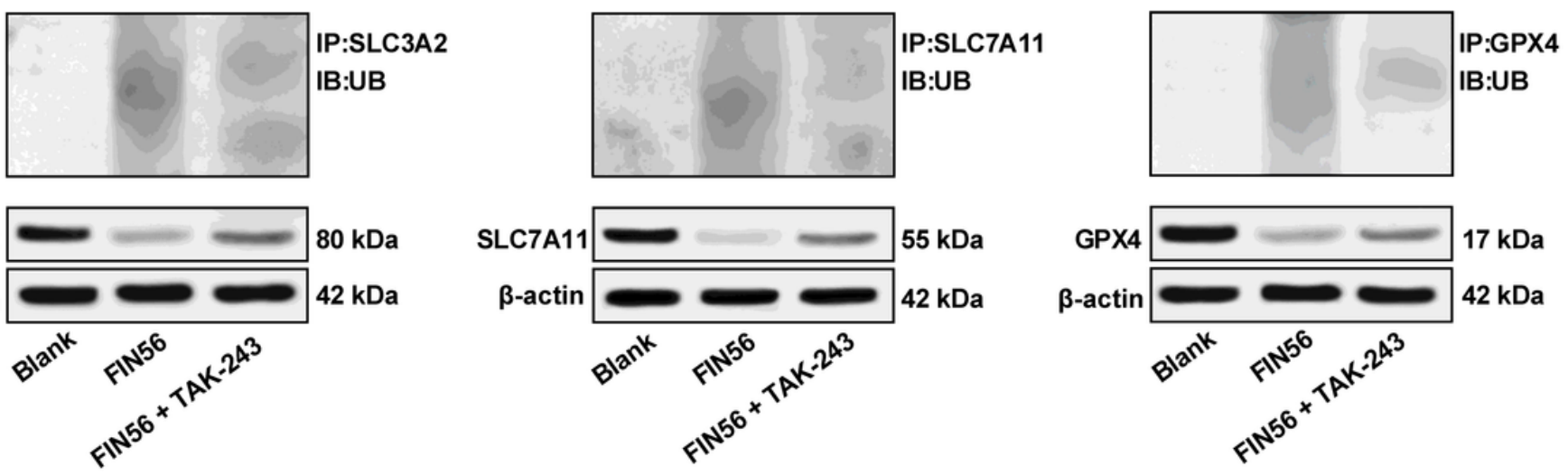

B
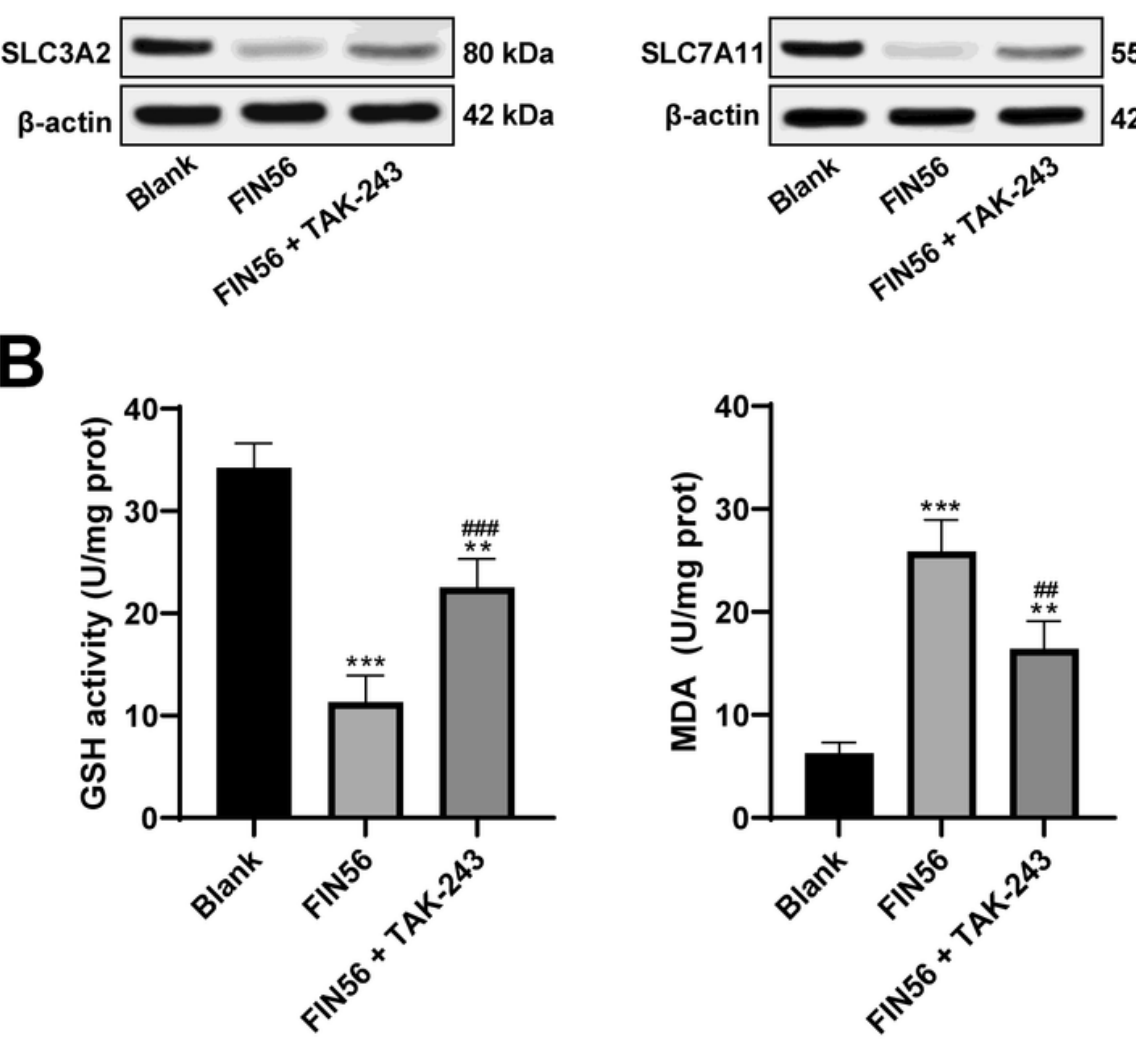

\section{Figure 6}

Inhibition of System Xc- and GPX4 ubiquitination in neurons reduced ferroptosis-induced injury. A, ubiquitination of SLC3A2, SLC7A11 and GPX4 in the brain of SAH rats was detected by Co-IP assay and western blot analysis; $\mathrm{B}, \mathrm{GSH}$ and MDA levels in the brain of SAH rats were detected by colorimetry. Compared with the blank group, $* * p<0.01$, $* \star * p<0.001$; compared with the FIN56 group, \#\# $p<0.01$, $\# \# \# p<0.001$. The data were analyzed using one-way ANOVA, followed by Tukey's multiple comparisons test. Replicates $=3$. 


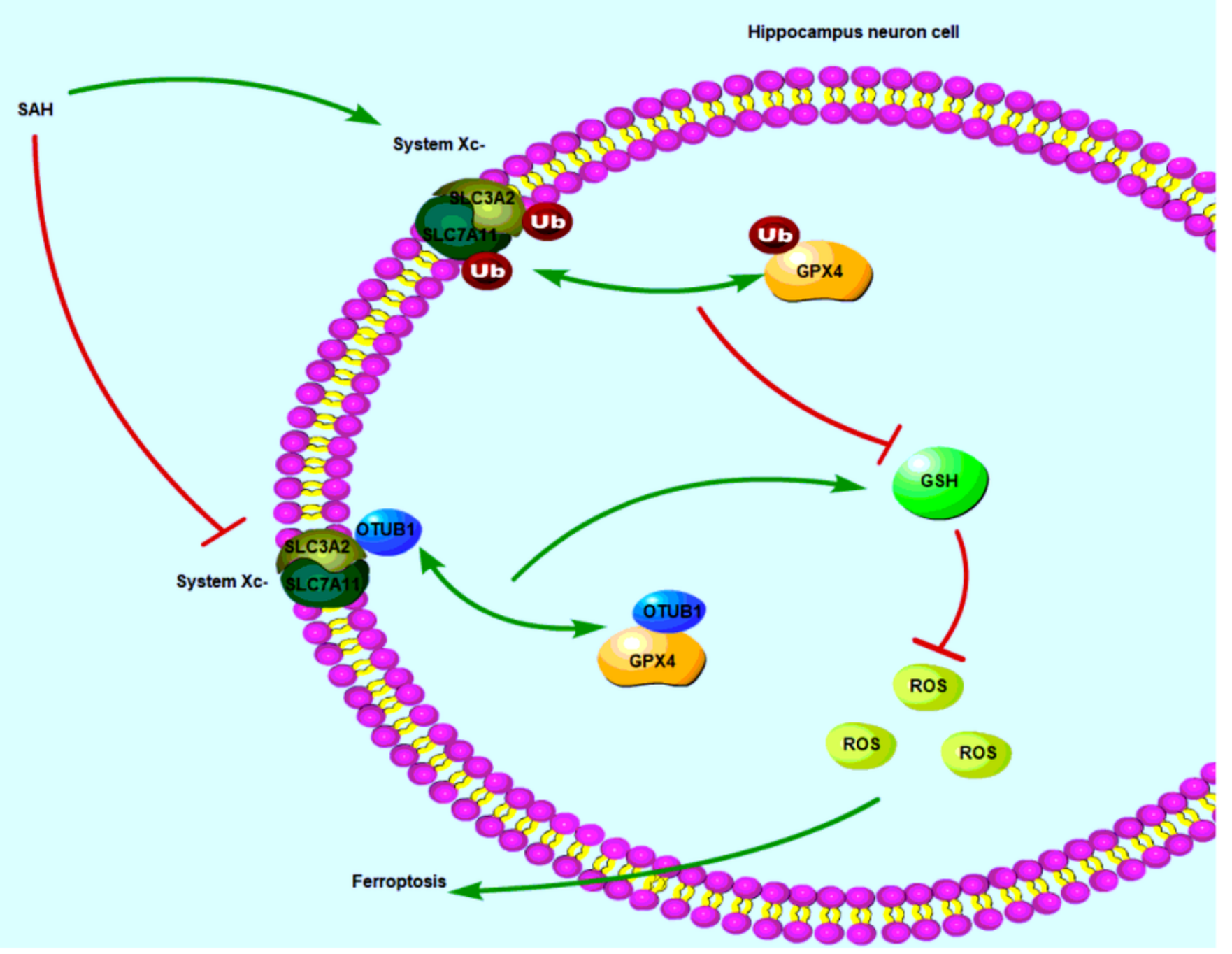

Figure 7

Mechanism of SAH induced ferroptosis in hippocampal neurons. SAH promoted the ubiquitination of System Xc- and GPX4, and inhibited the ubiquitination of OTUB1, SLC3A2 and GPX4, which led to GSH depletion, ROS accumulation and ferroptosis in neurons. 